\title{
The N-terminal domain of ALS-linked TDP-43 assembles without misfolding
}

Phoebe S. Tsoi ${ }^{1}$, Kyoungjae J. Choi $^{1}$, Paul G. Leonard ${ }^{2}$, Antons Sizovs ${ }^{1}$, Mahdi Muhammad Moosa $^{1}$, Kevin R. MacKenzie ${ }^{1,3}$, Josephine C. Ferreon ${ }^{1 \star}$, Allan Chris M. Ferreon ${ }^{1 \star}$ ${ }^{*}$ Corresponding authors

${ }^{1}$ Department of Pharmacology and Chemical Biology, Baylor College of Medicine, One Baylor Plaza, Houston, Texas, USA

${ }^{2}$ Department of Genomic Medicine and Core for Biomolecular Structure and Function, University of Texas MD Anderson Cancer Center, Houston, Texas, USA

${ }^{3}$ Department of Pathology and Immunology, Baylor College of Medicine, One Baylor Plaza, Houston, Texas, USA

*E-mail: allan.ferreon@bcm.edu; josephine.ferreon@bcm.edu

\section{Contributions:}

J.C.F. and A.C.F. designed the experiments; P.S.T. and A.C.F. performed and analyzed the ensemble CD and fluorescence spectroscopy, and single-molecule FRET experiments. P.S.T. and J.C.F. performed and analyzed the NMR experiments. K.J.C. prepared the TDP-43 DNA constructs and performed the imaging experiments. K.J.C., J.C.F. and A.C.F. purified and prepared the TDP-43 protein samples. P.G.L. carried out the analytical SEC and sedimentation velocity experiments. A.S. and J.C.F. performed the SEC-static light scattering experiments. K.R.M., M.M.M., J.C.F. and A.C.F wrote the manuscript, with inputs from all other authors. 


\section{Abstract}

TDP-43 forms inclusions in several neurodegenerative diseases, and both its $\mathrm{N}$ - and $\mathrm{C}$-terminal domains are implicated in this process. We show that the folded TDP-43 N-terminal domain oligomerizes under physiological conditions and propose that, in full-length TDP-43, association between folded $\mathrm{N}$-terminal domains enhances the propensity of the intrinsically unfolded $\mathrm{C}$ terminal domains to drive pathological aggregation. 
Amylotropic lateral sclerosis is a disorder of motor neurons that often results in a progressive fatal muscle atrophy ${ }^{1}$, and cytoplasmic TAR DNA-binding protein-43 (TDP-43) inclusion bodies are the salient features of both familial and sporadic disease pathologies ${ }^{2}$. Studies on the mechanism of TDP-43 action have revealed a complex interplay between the loss of cellular function and gain of toxic dysfunction effects of the TARDBP gene product ${ }^{1,3,4}$. TDP-43 comprises an N-terminal domain (TDP-43 ${ }^{\mathrm{NTD}}$ ), two RNA recognition motif single-stranded nucleic acid binding domains, and a disordered C-terminal domain (TDP- $43^{\mathrm{CTD}}$ ) ${ }^{5}$. The glycine rich C-terminal domain, which harbors the vast majority of disease-linked mutations ${ }^{4}$, selfinteracts and undergoes both liquid-liquid phase separation and aggregation ${ }^{6-8}$. While none of the hitherto identified pathogenic mutations reside within the $\mathrm{N}$-terminal domain, recent in vivo studies clearly indicate a role for the N-terminal domain in pathogenic aggregation ${ }^{3,9,10}$.

The TDP-43 N-terminal domain is highly conserved across the metazoan lineage ${ }^{11}$; although its physiological role remains elusive, it has been reported to self-associate in vitro ${ }^{12}$ and in cells ${ }^{9}$. Conflicting observations have been made about TDP-43 ${ }^{\text {NTD }}$ physical properties ${ }^{5,13,14}$. Qin et al ${ }^{13}$ report that a tagged construct of TDP-43 residues 1-102 adopts an unstable ubiquitin-like fold $\left(-30 \%\right.$ unfolded at $25^{\circ} \mathrm{C}, \mathrm{pH} 4.0$ in unbuffered water) and hypothesize that this unfolded state plays a role in initiating full-length TDP-43 aggregation. Mompeán et a $t^{5}$ find that a tagged construct of residues 1-77 is completely folded and monomeric under similar solution conditions $\left(25^{\circ} \mathrm{C}, \mathrm{pH} 3.8,3 \mathrm{mM}\right.$ sodium acetate). The contradictory findings may arise from sample conditions, construct lengths, expression tags ${ }^{14}$, or expression/purification methods. Here, we integrate observations from ensemble biophysical techniques and single-molecule methods to assess TDP-43 ${ }^{\text {NTD }}$ stability and self-association, focusing on near-physiological conditions but also examining a wide $\mathrm{pH}$ range to attempt to reconcile divergent literature reports.

Despite reports of poor TDP-43 ${ }^{\text {NTD }}$ in vitro solution properties ${ }^{13}$, we find refolded TDP-43 ${ }^{\text {NTD }}$ (residues 1-80, no tags) to be readily soluble and suitable for ensemble experiments at nearphysiological conditions ( $\mathrm{pH} 7.5,200 \mathrm{mM} \mathrm{NaCl}$ ). Thermal denaturation of TDP-43 ${ }^{\mathrm{NTD}}$ ( $\alpha \beta \mathrm{Y}$ buffer, pH $7.5 \pm 0.05$ ) show clear pre-transition baselines (Fig. 1a), indicating that the native folded state is the major ensemble species. To probe for minor species that might escape detection in ensemble-averaged experiments, and to eliminate any self-association effects, we used single-molecule Förster resonance energy transfer (smFRET) experiments that can directly resolve minor species in a mixture ${ }^{15}$. We labeled TDP-43 ${ }^{\text {NTD }}$ with donor and acceptor dyes (Alexa Fluor 488 \& Alexa Fluor 594; see Supplementary Methods) at residues 2 and 81, which are close in space in the folded domain (PDB $2 n 4 p^{5}$ ). As a single doubly-labeled protein in the dilute sample (100 pM) traverses through the femtoliter-observation volume, we record fluorescence signals from the donor and acceptor dyes. Signal intensities from individual channels are analyzed to yield FRET efficiency $\left(E_{\mathrm{FRET}}\right)$ histograms. Folded, doubly labeled TDP$43^{\mathrm{NTD}}$ gives high $E_{\mathrm{FRET}}$ values (Fig. 1c, top panel); decreased $E_{\mathrm{FRET}}$ implies increased distance between the dyes, from which we infer TDP-43 ${ }^{\text {NTD }}$ unfolding and expansion. Near-zero $E_{\text {FRET }}$ values (gray shaded region at left in Fig. 1c) arising from TDP-43 ${ }^{\text {NTD }}$ molecules with absent, photobleached, or otherwise nonfluorescent acceptor dyes are excluded from our analysis.

Binned SmFRET data in the absence of denaturant are consistent with a single-distribution $E_{\text {FRET }}$ value of $\sim 0.85$, and we infer the existence of a single, folded population at $\mathrm{pH} 7.5$ (Fig. 1c, 
top). Chemical denaturation with guanidine hydrochloride $(\mathrm{GdnHCl})$ lowers $E_{\mathrm{FRET}}$ (with $\sim 1 \mathrm{M}$ $\left.\mathrm{GdnHCl}, E_{\mathrm{FRET}} \approx 0.65\right)$. At intermediate denaturant concentrations, histograms reveal coexisting $E_{\mathrm{FRET}}$ distributions for both folded and unfolded protein states (Fig. 1c, panels 2-4 from top), indicating slow interconversion between the states on the experimental time scale ( 500 $\mu \mathrm{s})$. The native state mean $E_{\mathrm{FRET}}$ remains relatively unchanged from 0 to $2 \mathrm{M}$ denaturant; we infer that the native state does not expand significantly with denaturant but is depopulated in favor of the unfolded state. In contrast, the denatured ensemble mean $E_{\mathrm{FRET}}$ decreases at elevated $\mathrm{GdnHCl}$ (Fig. 1c and Supplementary Fig.13), similar to denaturant-induced expansion of unfolded ensembles of globular proteins ${ }^{16,17}$.

The TDP- $43^{\mathrm{NTD}}$ thermal transition midpoint for unfolding $\left(T_{\mathrm{m}}\right)$ is higher than $50^{\circ} \mathrm{C}$ from $\mathrm{pH} 6$ to 8 but drops dramatically with extremes of $\mathrm{pH}$ (Fig. 1b and Supplementary Fig. 7). The steep dependence of $T_{\mathrm{m}}$ on $\mathrm{pH}$ under acidic conditions may explain how experiments using different constructs and buffer conditions access the unfolded state to different extents. At nearphysiological salt and $\mathrm{pH}$ conditions, however, our ensemble and single-molecule data establish that TDP $-43^{\text {NTD }}$ is folded from low $\mathrm{pM}$ to $\mu \mathrm{M}$ concentrations. Successive rounds of thermal unfolding and refolding without detectable precipitation and near identical $T_{\mathrm{m}}$ 's show that TDP$43^{\mathrm{NTD}}$ folding is robustly reversible near physiological $\mathrm{pH}$ and salt.

Because our refolded TDP-43 ${ }^{\mathrm{NTD}}$ is stable and soluble over a broad $\mathrm{pH}$ range, we used NMR spectroscopy to probe its structure. ${ }^{1} \mathrm{H}^{-15} \mathrm{~N}$ TROSY-HSQC spectra of deuterated TDP-43 ${ }^{\mathrm{NTD}}$ samples (Supplementary Figs. 10-12) at pH 4.0 and zero salt show well-dispersed peaks with shifts similar to those reported by Mompeán et $\mathrm{al}^{5}$, indicating that our TDP-43 ${ }^{\mathrm{NTD}}$ adopts a fold similar to their construct despite lacking a His tag and having four additional C-terminal residues. However, our TDP-43 ${ }^{\mathrm{NTD}}$ spectra at $\mathrm{pH} 7.0$ and $50 \mathrm{mM} \mathrm{NaCl}$ differ strongly from those of Mompeán et $\mathrm{al}^{5}$ : although the amides are well-dispersed, only half of the expected resonances are visible at $25^{\circ} \mathrm{C}$ (Fig. 2a and Supplementary Fig. 10a and 11). Raising the temperature to $40^{\circ} \mathrm{C}$ and lowering the protein concentration increases the number of peaks but also their heterogeneity (Supplementary Figs. 11 and 12). However, we were able to assign the backbone chemical shifts of the domain as a $\mathrm{H}_{6}$ GB1-TDP-43 ${ }^{\mathrm{NTD}}$ fusion $(\sim 20 \mathrm{kDa})$ at $\mathrm{pH} 6.8$ (Supplementary Table 1). The backbone shifts are similar ${ }^{5}$ to those reported for the isolated domain at pH 4 (Supplementary Fig. 10b), indicating a similar fold. We propose that TDP$43^{N T D}$ self-association at neutral $\mathrm{pH}$ broadens resonances for amides positioned near the protein-protein interface, and that both low $\mathrm{pH}$ and $\mathrm{N}$-terminal fusions decrease self-association. Resonances that are assigned in the fusion protein but absent in our spectra of the isolated domain at $\mathrm{pH} 7.0$ map to a contiguous surface in TDP-43 ${ }^{\mathrm{NTD}}$ corresponding to the last $3 \beta$ strands (Fig. 2b), supporting our proposal.

To test directly for self-association, we performed sedimentation velocity analytical ultracentrifugation (AUC) and size-exclusion chromatography (SEC) analyses of TDP-43 ${ }^{\mathrm{NTD}}$ dilution series. Both AUC and SEC show that TDP-43 ${ }^{\text {NTD }}$ exists as monomer, dimer, and tetramer species, but not as large aggregates (Fig. 2c-e and Supplementary Figs. 8 and 9); the smallest SEC species is determined to be a monomer by static light scattering (Supplementary Fig. 8b). The higher order species readily dissociate to monomer and dimer, consistent with 
equilibrium self-association and in contrast to amyloidogenic proteins that aggregate irreversibly ${ }^{18}$.

Ensemble thermal unfolding fluorescence measurements show that the TDP-43 ${ }^{\text {NTD }}$ thermal unfolding mid-point temperature $\left(T_{\mathrm{m}}\right)$ increases with protein concentration (Supplementary Figs. 2-4). Because this finding contradicts the concentration-dependent unfolding described by Qin et al. ${ }^{13}$, we performed smFRET on doubly-labeled 100 pM TDP-43 ${ }^{\text {NTD }}$ titrated with up to 120 $\mu \mathrm{M}$ unlabeled TDP-43 ${ }^{\mathrm{NTD}}$ (Supplementary Fig. 14). The folded state $E_{\mathrm{FRET}}$ peak is not significantly perturbed and no minor species are detected across this $10^{6}$-fold change in protein concentration, so we conclude that self-associating TDP-43 ${ }^{\text {NTD }}$ maintains its global fold. Together, these data establish that the TDP-43 N-terminal domain self-associates under physiological buffer conditions through a folded, native state. The conservation of the TDP$43^{\text {NTD }}$ sequence ${ }^{11}$ suggests that oligomerization is important to TDP-43 cellular function, perhaps by modulating the affinity of the TDP-43 RNA recognition motifs for nucleic acids ${ }^{12}$.

Although the cellular function of TDP-43 ${ }^{\mathrm{NTD}}$-mediated assembly may not be fully elucidated at present, some key implications for protein dysfunction and misfolding are clear. The intrinsically disordered TDP-43 C-terminal domain (TDP-43 ${ }^{\mathrm{CTD}}$ ) is proposed to be the 'amyloidogenic core' that drives TDP-43 aggregation ${ }^{7,19}$; at $45 \mu \mathrm{M}, \sim 8 \%$ of TDP- $43^{\mathrm{CTD}}$ can initiate assembly, resulting in phase separation ${ }^{7}$. Here, we have shown that TDP- $43^{\mathrm{NTD}}$ forms reversible oligomers to a greater extent at lower concentrations ( $-30 \%$ dimer at $2-5 \mu \mathrm{M} ; \sim 10 \%$ tetramer at $20 \mu \mathrm{M}$; see Supplementary Fig. 9). We propose that, under physiological conditions, full-length TDP-43 can assemble reversibly to dimers and tetramers via the folded TDP-43 ${ }^{\mathrm{NTD}}$, and that these oligomers enhance the rate of TDP- $43^{\mathrm{CTD}}$-mediated liquid-liquid phase separation, irreversible aggregation and/or fibril formation by raising the effective local concentration of the C-terminal domains relative to one another (Fig. 3). Our model explains the importance of TDP-43 ${ }^{\mathrm{NTD}}$ in driving a fusion protein comprising N-terminal and C-terminal TDP-43 domains flanking GFP to form giant liquid droplets with a critical concentration of phase separation of $\sim 5 \mu \mathrm{M}^{20}$. The possibility that TDP-43 ${ }^{\mathrm{NTD}}$ itself might directly favor phase separation at physiological $\mathrm{pH}$ is at odds with the data presented here and is directly rebutted by its failure to do so under conditions where TDP- $43^{\text {CTD }}$ readily phase separates and forms fibrils (Supplementary Fig. 15). Our model also explains why a minimal TDP-43 construct lacking RRM domains requires the TDP$43 \mathrm{~N}$-terminal domain to induce aggregation of endogenous TDP- $43^{21}$ : the $\mathrm{N}$-terminal domain within the artificial construct helps recruit the endogenous protein through its $\mathrm{N}$-terminal domain. TDP-43 may represent a natural example of the concept demonstrated in an artificial construct by Shin et al. ${ }^{22}$, where the light-inducible oligomerization domain of Cry2 dramatically accelerates liquid-liquid phase separation of a fusion protein containing a disordered domain in a light-dependent manner. Furthermore, N-terminal self-assembly may also explain why TDP-43 is found in pathological inclusions with other proteins that contain intrinsically disordered domains ${ }^{23}$ : if a single TDP-43 were incorporated into an aggregate via its C-terminal domain, it could recruit more TDP-43 through its N-terminal domain.

Other biophysical work has suggested that the unfolded state of TDP-43 ${ }^{\text {NTD }}$ contributes to aggregation during cellular dysfunction ${ }^{13}, 14$. Although TDP- $43^{\text {NTD }}$ can access the unfolded state readily at extremes of $\mathrm{pH}$, we have shown that under near-physiological conditions there are no 
detectable misfolding intermediates by which TDP-43 ${ }^{\mathrm{NTD}}$ itself might aggregate. Instead, the stabe, folded N-terminal domain promotes TDP-43 ${ }^{\mathrm{CTD}}$-driven TDP-43 aggregation without itself misfolding, by mediating TDP-43 oligomerization.

\section{Acknowledgements}

We thank Jin Wang's laboratory for the use of the SEC-SLS equipment. We thank Debra Townley and Baylor College of Medicine Microscopy Core for the TEM data collection. This work was supported by laboratory startup funds from Baylor College of Medicine (A.C.F. and J.C.F.). The authors declare no competing financial interests. 

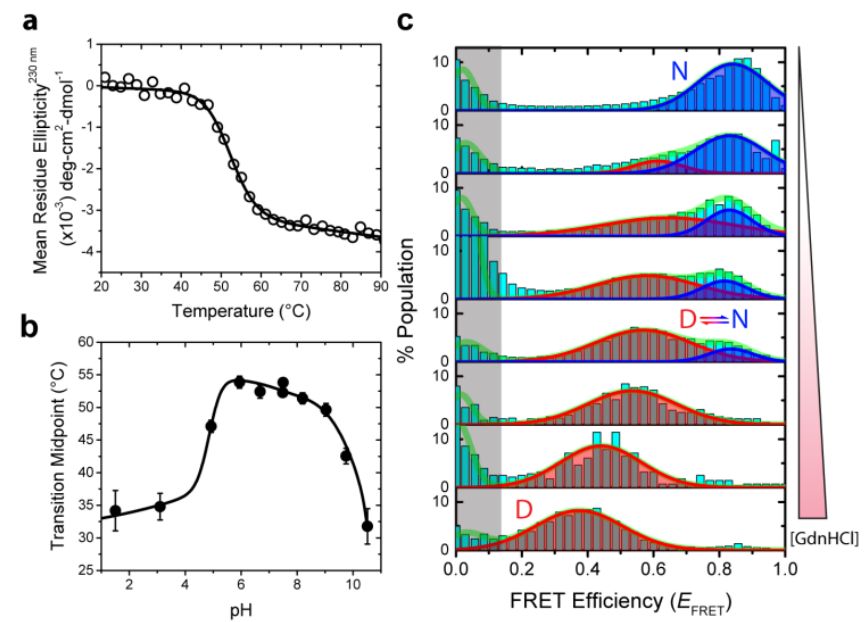

Figure 1. TDP $-43^{\mathrm{NTD}}$ is thermodynamically stable at physiological conditions. (a) Thermal unfolding of TDP-43 ${ }^{\text {NTD }}$ was monitored via far-UV CD spectroscopy. Data is shown as open circles; non-linear least squares (NLS) fit of the data to a two-state protein-denaturation model is shown as a line. Transition midpoint(s) are obtained from the NLS fit. (b) Changes in transition midpoints as a function of $\mathrm{pH}$ showing TDP-43 ${ }^{\mathrm{NTD}}$ is thermodynamically stable at physiological conditions and access acid- and alkaline-unfolded states at respective $\mathrm{pH}$ conditions. $T_{\mathrm{m}}$ values are shown as filled circles; the line represents equi-population boundary of folded and unfolded states as determined through global phase diagram analysis. See also Supplementary Fig. 7. (c) Single-molecule FRET histograms of two-state unfolding of TDP-43 ${ }^{\text {NTD }}$ upon chemical denaturation. The protein shows no detectable misfolding intermediate and at intermediate denaturant concentration undergoes slow $\mathrm{N} \rightleftharpoons \mathrm{D}$ interconversion within the temporal resolution of our experiments $(500 \mu \mathrm{s})$. GdmHCl concentrations for individual panels are: $0,1,1.25,1.5$, $1.75,2,4$, and $6 \mathrm{M}$ (top to bottom). 
a

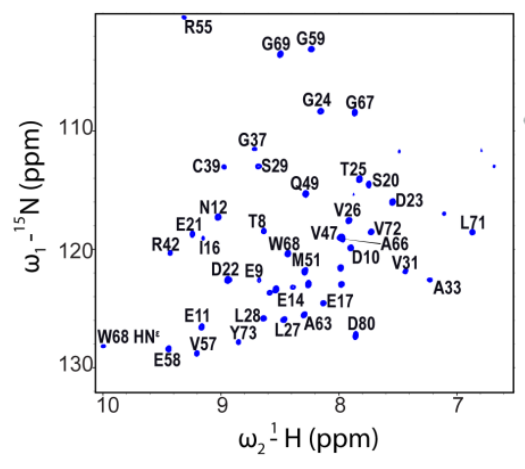

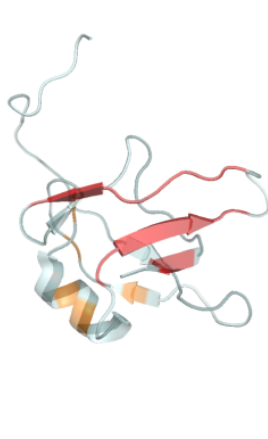

c

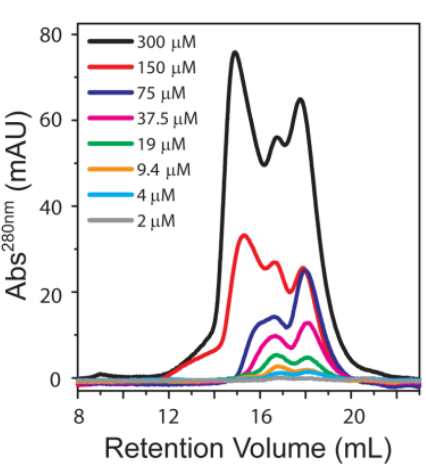

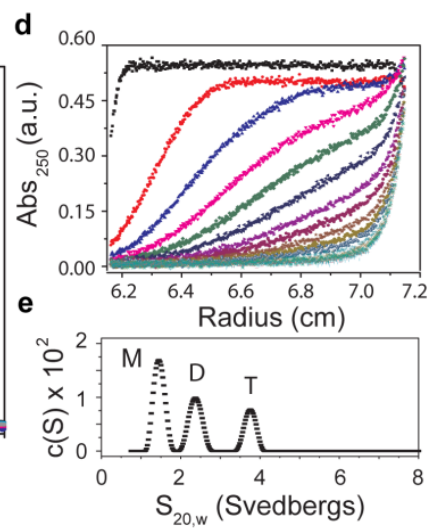

Figure 2. Reversible assembly of TDP- $43^{\mathrm{NTD}}$. (a) ${ }^{1} \mathrm{H}-{ }^{15} \mathrm{~N}$ TROSY-HSQC spectra of TDP-43 ${ }^{\mathrm{NTD}}$ showing dispersed peaks, albeit with several missing resonances. The missing resonances map to a contiguous surface in TDP-43 ${ }^{\mathrm{NTD}}\left[(\mathbf{b})\right.$, red color; PDB: $\left.2 n 4 \mathrm{p}^{5}\right]$. Residues that sample alternate states are shown in orange (see Supplementary Figure $\mathbf{1 1}$ for details). Size exclusion chromatography (SEC) elution profiles are shown in (c) for samples containing 2-300 $\mu \mathrm{M}$ TDP$43^{\text {NTD }}$ at physiological buffer conditions. Sequential two-fold dilution of $300 \mu \mathrm{M}$ TDP-43 ${ }^{\text {NTD }}$ resulted in dissociation of the high MW species (tetramer, T) to lower dimer (D) and monomeric (M) species, indicating a reversibly oligomerizing system that contrasts irreversible fibril formation ${ }^{18}$. Sedimentation boundaries (d) and $c(S)$ distributions (e) for $37 \mu \mathrm{M}$ TDP-43 ${ }^{\mathrm{NTD}}$ from sedimentation velocity analytical ultracentrifugation experiment shows self-association in a concentration-dependent fashion (see Supplementary Figure 9 for details). 


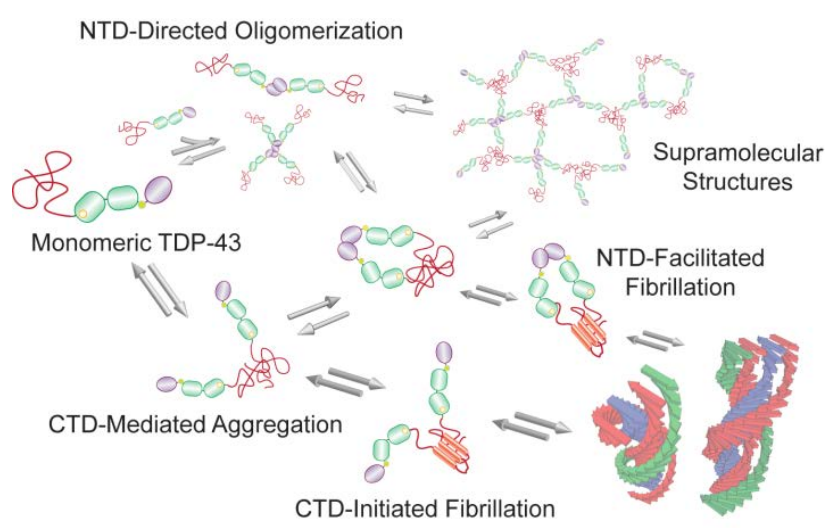

Figure 3. Model for TDP-43 N-terminus- facilitated C-terminal assembly and aggregation. TDP-43 comprises a fully-folded TDP-43 ${ }^{N T D}$ (dark blue) that oligomerizes, two RNA binding domains (light green), and a C-terminal domain (TDP- $43^{\mathrm{CTD}}$, red) that can convert from unstructured to folded intermediate and amyloid fibril states. The N-terminus can facilitate TDP$43^{\text {CTD }}$ phase transitions as well as C-terminus interactions that can lead to TDP-43 fibril formation or pathological aggregation. 


\section{References}

1. Robberecht, W. \& Philips, T. The changing scene of amyotrophic lateral sclerosis. Nat Rev Neurosci 14, 248-264 (2013).

2. Andersen, P.M. \& Al-Chalabi, A. Clinical genetics of amyotrophic lateral sclerosis: what do we really know? Nat Rev Neurol 7, 603-615 (2011).

3. Zhang, Y.-J. et al. Aberrant cleavage of TDP-43 enhances aggregation and cellular toxicity. Proc Natl Acad Sci U S A 106, 7607-7612 (2009).

4. Lee, E.B., Lee, V.M.Y. \& Trojanowski, J.Q. Gains or losses: molecular mechanisms of TDP43-mediated neurodegeneration. Nat Rev Neurosci 13, 38-50 (2012).

5. Mompeán, M. et al. The TDP-43 N-terminal domain structure at high resolution. FEBS J 283, 1242-1260 (2016).

6. Wang, I.-F. et al. The self-interaction of native TDP-43 C terminus inhibits its degradation and contributes to early proteinopathies. Nat Commun 3, 766 (2012).

7. Conicella, A.E., Zerze, G.H., Mittal, J. \& Fawzi, N.L. ALS Mutations Disrupt Phase Separation Mediated by alpha-Helical Structure in the TDP-43 Low-Complexity CTerminal Domain. Structure 24, 1537-1549 (2016).

8. Kitamura, A. et al. Interaction of RNA with a C-terminal fragment of the amyotrophic lateral sclerosis-associated TDP43 reduces cytotoxicity. Sci Rep 6, 19230 (2016).

9. Zhang, Y.-J. et al. The dual functions of the extreme N-terminus of TDP-43 in regulating its biological activity and inclusion formation. Hum Mol Genet 22, 3112-3122 (2013).

10. Budini, M., Romano, V., Quadri, Z., Buratti, E. \& Baralle, F.E. TDP-43 loss of cellular function through aggregation requires additional structural determinants beyond its Cterminal Q/N prion-like domain. Hum Mol Genet 24, 9-20 (2015).

11. Wang, H.-Y., Wang, I.F., Bose, J. \& Shen, C.K.J. Structural diversity and functional implications of the eukaryotic TDP gene family. Genomics 83, 130-139 (2004).

12. Chang, C.K. et al. The N-terminus of TDP-43 promotes its oligomerization and enhances DNA binding affinity. Biochem Biophys Res Commun 425, 219-224 (2012).

13. Qin, H., Lim, L.Z., Wei, Y. \& Song, J. TDP-43 N terminus encodes a novel ubiquitin-like fold and its unfolded form in equilibrium that can be shifted by binding to SSDNA. Proc Natl Acad Sci U S A 111, 18619-18624 (2014).

14. Chang, C.K. \& Huang, T.H. Untangling the structure of the TDP-43 N-terminal domain. FEBS J 283, 1239-1241 (2016).

15. Schuler, B. \& Eaton, W.A. Protein folding studied by single-molecule FRET. Curr Opin Struct Biol 18, 16-26 (2008).

16. Merchant, K.A., Best, R.B., Louis, J.M., Gopich, I.V. \& Eaton, W.A. Characterizing the unfolded states of proteins using single-molecule FRET spectroscopy and molecular simulations. Proc Natl Acad Sci U S A 104, 1528-1533 (2007).

17. Ziv, G., Thirumalai, D. \& Haran, G. Collapse transition in proteins. Phys Chem Chem Phys 11, 83-93 (2009).

18. Fink, A.L. Protein aggregation: folding aggregates, inclusion bodies and amyloid. Fold Des 3, R9-23 (1998).

19. Jiang, L.L. et al. Two mutations G335D and Q343R within the amyloidogenic core region of TDP-43 influence its aggregation and inclusion formation. Sci Rep 6, 23928 (2016).

20. Schmidt, H.B. \& Rohatgi, R. In Vivo Formation of Vacuolated Multi-phase Compartments Lacking Membranes. Cell Rep 16, 1228-1236 (2016).

21. Romano, V., Quadri, Z., Baralle, F.E. \& Buratti, E. The structural integrity of TDP-43 Nterminus is required for efficient aggregate entrapment and consequent loss of protein function. Prion 9, 1-9 (2015).

22. Shin, Y. et al. Spatiotemporal Control of Intracellular Phase Transitions Using LightActivated optoDroplets. Cell 168, 159-171 e114 (2017). 
bioRxiv preprint doi: https://doi.org/10.1101/134072; this version posted May 4, 2017. The copyright holder for this preprint (which was not certified by peer review) is the author/funder, who has granted bioRxiv a license to display the preprint in perpetuity. It is made available under aCC-BY-NC-ND 4.0 International license.

23. Villemagne, V.L., Fodero-Tavoletti, M.T., Masters, C.L. \& Rowe, C.C. Tau imaging: early progress and future directions. Lancet Neurol 14, 114-124 (2015). 